\title{
Cancer stem cells modulate patterns and processes of evolution in cancers
}

Lucie Laplane $e^{1,2}$

1. UMR8590 Institut d'Histoire et Philosophie des Sciences et des Techniques (IHPST), CNRS, Université Paris I Panthéon-Sorbonne, ENS, 13 rue du Four, 75006 Paris, France;

2. UMR1170, Inserm, Gustave Roussy, 114 rue Edouard Vaillant, Villejuif, France

\section{Correspondence to}

Lucie Laplane

IHPST, Université Paris I Panthéon-Sorbonne.

13 rue du Four

75006 Paris, France

e-mail: lucie.laplane@univ-paris1.fr

tel: $+33(0) 143546036$

fax: +33(0)143252948

\section{Abstract}

The clonal evolution (CE) model and the cancer stem cell (CSC) model are two independent models of cancers, yet recent data shows intersections between the two models. This article explores the impacts of the CSC model on the CE model. I show that CSC restriction, which depends on CSC frequency in cancer cell populations and on the probability of dedifferentiation of cancer non-stem cells (non-CSC) into CSCs, can favor or impede some patterns of evolution (linear or branched evolution) and some processes of evolution (drift, evolution by natural selection, complex adaptations). Taking CSC restriction into account for the CE model thus has implications for the way in which we understand the patterns and processes of evolution, and can also provide new leads for therapeutic interventions.

\section{Key words}

Cancer stem cell; Clonal evolution; Cancer progression; Drift; Evolution by natural selection

\section{Acknowledgments}

This work was financed by the program "Stratified Oncology Cell DNA Repair And Tumor Immune Elimination" (SOCRATE) of the Site of Integrated Research In Cancerology (SIRIC) in Gustave Roussy (grant INCa-DGOS-INSERM 6043) and the Cancéropôle île-de-France (grant 2016-1-EMERG-50-CNRS DR 1-1).

I am deeply grateful to the reviewers. I have benefited from the best reviews possible and their remarks and critiques were particularly helpful to revise and strengthen my argument. Kate MacCord did an amazing job not only with editing the English but also with helping me to clarify my arguments. This paper also greatly benefited from many comments from philosophers, biologists, and oncologists, on its early and/or later versions. I am particularly grateful to Melinda Fagan, Jean Gayon, Pierre-Luc Germain, Peter Godfrey-Smith, Kate MacCord, Francesca Merlin, Thomas Pradeu, Murielle Gaudry, Stéphane Giraudier, Anne-Marie Lyne, Mrinal Patnaik, Leïla Perié, and especially to Eric Solary and all of my team at Gustave Roussy. 
The clonal evolution (CE) model and the cancer stem cell (CSC) model are two models of cancer that have an ambiguous theoretical relationship. The CE model describes cancer as an evolutionary process in which the accumulation of genetic and epigenetic alterations results in the diversification of cancer cells through space and time (Figure 1a). The CSC model states that cancers mimic tissue organization and emerge and develop only from a restricted fraction of cancer cells with stem-like properties called CSCs (Figure 1b). Historically, the CSC model was framed in opposition to the CE model (Reya et al. 2001; Wicha et al. 2006; Shipitsin et al. 2007; Pantic 2011). Some investigators have since suggested that the two competing models are "not necessarily mutually exclusive" (Polyak 2007, 3160; Campbell and Polyak 2007; Lagasse 2008), that one or the other could more adequately explain different types of cancer (Adams and Strasser 2008; Shackleton et al. 2009), or that the two models are complementary and should be combined because they each provide explanations of different aspects of the same cancer (Fabian et al. 2009; Greaves 2010; Lang et al. 2015).

Several lines of experimentation have shown that CE occurs in CSCs (for the first evidence see Piccirillo et al. 2009; Anderson et al. 2011; Notta et al. 2011). Taking into account the impact of CE on CSCs in the CSC model appears to be important for at least two reasons. First, for the therapeutic strategy that emerged from the CSC model and that consists of specifically targeting the CSCs during cancer treatments, it means that CSCS can represent a heterogeneous target. CSCs can differ both synchronically (at the diagnosis for example) and diachronically (from diagnosis to relapse for example). This heterogeneity between CSCs can result in the coexistence of CSCs with various sensitivities to therapies (e.g. Meyer et al. 2015). Second, the accumulation of new mutations in CSCs can modify their properties. For example, additional mutations could lead to the acquisition of migratory ability, distinguishing "stationary CSCs" from "migrating CSCs" able to generate metastases (Brabletz et al. 2005; Bapat 2007; Odoux et al. 2008; Takebe and Ivy 2010). By not taking into account $\mathrm{CE}$, the CSC model misses the heterogeneity of this CSC population. The incorporation of CE into the CSC model is now fairly accepted (e.g. Kreso and Dick 2014).

In contrast, the CE model still largely ignores the CSC model. Throughout this paper, I argue that CSCs impact CE, particularly through a phenomenon that I call "CSC restriction" (referring to the fact that CSCs are only a restricted fraction of a given cancer), and thus that the CE model needs to incorporate some features of the CSC model in order to account for all of the consequences that CSC restriction has for CE.

This research took root from previous philosophical works. In his book on Darwinian populations, Godfrey-Smith (2009) introduced a framework that allows one to distinguish different types of Darwinian populations, for example paradigmatic Darwinian populations in which evolution by natural selection can produce complex adaptations, i.e. novel traits, versus marginal Darwinian populations in which evolution by natural selection can only produce simple adaptations, i.e. change in trait frequency. Distinguishing between types of Darwinian populations depends on various parameters of evolution that can be roughly measured (among which are the classical requirements of evolution by natural selection such as variation, heritability, and differential fitness, as well as additional ones that can also modify how Darwinian a population is). Applying this framework to cancers, Germain (2012) highlighted that cancer cell populations are much less like paradigmatic Darwinian populations than usually assumed and this is in large part due to the CSC model, which appears as a major factor that limits the ability of cancer cells to evolve by natural selection (see section 1.1). Germain's conclusion was the point of 
departure for my work. If cancer cells are much less like paradigmatic Darwinian populations than expected, then what kind of populations are they exactly? In this paper, I build a framework similar to Godfrey-Smith's in which the measure of some factors associated with CSCS changes the CE features of cancer cell populations. The two factors of interest are CSC frequency (how many cancer cells are CSCs?) and non-CSC dedifferentiation (what is the probability of non-CSCs becoming CSCs?), which capture what I call "CSC restriction". The CE features under the influence of CSC restriction are processes of evolution (evolution by natural selection versus neutral evolution, complex versus simple adaptations) and patterns of evolution (linear versus branched evolution).

The first section shows that CSC restriction limits evolution by natural selection (and more generally CE), prevents complex adaptations, and favors drift and linear evolution. Scientists and philosophers have compared CSC restriction to other phenomena well known in evolutionary biology such as reproductive specialization or effective population size, raising the question of whether we could describe the impact of CSC restriction on CE through classical parameters of evolution. The second section shows that none of these parameters accurately account for CSC restriction, in particular because it relies on two different factors (CSC frequency and probability of dedifferentiation). The last section explores the potential biomedical interests of investigating the impact of CSC restriction on patterns of CE.

a. CE model

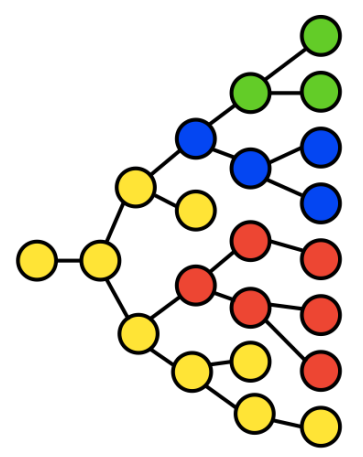

$\bigcirc$ Subclones with different mutations

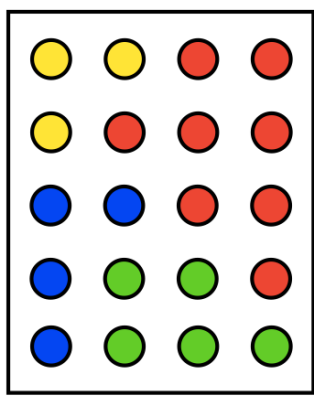

\section{b. CSC model}
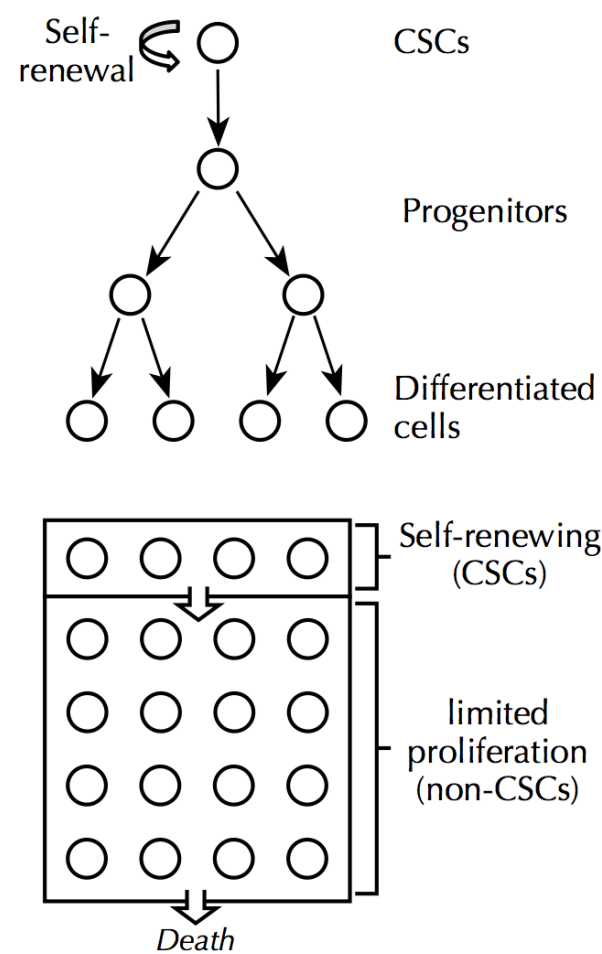

Figure 1 The CE model and the CSC model. To compare the CE model and the CSC model, I propose two classical representations of the models ( $a$ and $b$, top panels) and two more abstracted representations of these models ( $a$ and $b$, bottom panels). The more abstracted representations are useful to represent graphically the consequence of CSC restriction on CE in the next figure 


\section{CSC restriction impacts CE}

Before exploring the impact of CSCS on CE it is necessary to have a clear understanding of CSCs, and in particular of what I call CSC restriction. The CSC model pictures cancers as a caricature of the normal tissues from which they emerge (for a recent discussion, see Batlle and Clevers 2017). In most normal tissues in homeostasis, there is a hierarchical organization of cells in which a small fraction of stem cells sit at the apex of a hierarchy of differentiating cells. When stem cells divide, they can give rise to new stem cells, thus ensuring the maintenance of the stem cell pool, and/or they can differentiate into more mature cells (Fig 1b). In cancers, this organization is altered (differentiation can be blocked, biased, or amplified), yet in many cases, a hierarchy is maintained and only a fraction of the cancer cells (the CSCs) is able to develop and maintain the tumor. ${ }^{1}$

The explanatory value of the CSC model relies on two premises: first that not all cancer cells are CSCs, and second, that CSCs can be distinguished from non-CSCs (i.e. the observed functional difference between CSCs and nonCSCs is not stochastic). The massive search to identify CSCs in all cancers has produced conflicting data for both premises. First, estimated CSC frequency can vary by orders of magnitude, from very rare in some cancers (e.g. 50 in $10^{6}$ in acute myeloid leukemia; Bonnet and Dick 1997) to very common in others (e.g. 1 out of 4 cells in advanced melanoma; Quintana et al. 2008). Some mutations can result in an increase in CSC frequency, indicating that CSC frequency can also change during cancer progression (Notta et al. 2011; Clappier et al. 2011). Thus, the CSC frequency varies depending on cancer types and stages, and it can be measured, at least roughly. ${ }^{2}$ Second, in some cancers, non-CSCs can dedifferentiate into CSCS (e.g. Chaffer et al. 2013; Koren et al. 2015; Van Keymeulen et al. 2015; Medema 2017). Dedifferentiation affects the quantification of the cancer cells that contribute to cancer maintenance and evolution through time because when dedifferentiation is possible, in addition to the actual CSCs, the non-CSCs represent a pool of potential CSCs. How many of these non-CSCs will become CSCs is a variable that can be measured as the probability of dedifferentiation. Dedifferentiation of nonCSCs into CSCs is a process specific to some cancers (mostly observed in epithelial cancers and never observed in blood cancers so far) and the probability of dedifferentiation of a non-CSC into a CSC can vary between cancers (e.g. between breast cancers from different cell of origin, Latil et al. 2017).

I use the term 'CSC restriction' to refer to these two dynamic aspects of CSCS (CSC frequency, e.g. their actual number, and non-CSC dedifferentiation, e.g. the probability that non-CSCs dedifferentiate into CSCs). The level of CSC restriction thus varies in different cancer types and/or stages. CSC restriction is high when the CSC frequency and the probability of dedifferentiation are low and vice-versa. This section discusses the impact of the level of CSC restriction on CE, based on philosophical, modeling, and biological literature.

\subsection{CSC restriction limits evolution by natural selection and prevents complex adaptations}

\footnotetext{
${ }^{1}$ The cell of origin can either be a stem cell transformed into a CSC or a non-stem cell that reacquired stemness at transformation and became a CSC. For a detailed account of the history, structure and content of the CSC model, see Laplane (2014, 2016, chap 2-4).

${ }^{2}$ CSC quantification relies on many experimental parameters that can all introduce biases (reviewed in Kreso and Dick 2014; Nassar and Blanpain 2016).
} 
In the CE model, all the cancer cells can, in principle, evolve. Cancers are populations of cells that asexually reproduce through cell division. During division they can acquire mutations that they transmit to their daughter cells. The impact of mutations on cell fitness is variable so that two types of mutations are usually distinguished in cancers: passenger mutations, i.e. those that do not change the fitness of the cells, and driver mutations, i.e. those that increase their fitness (a mutation can be passenger in one context and driver in another). Cancer cells thus fulfill the minimal requirements for evolution by natural selection; they are populations of cells that reproduce with some heritable variation in fitness (Lewontin 1970; 1978). Taking the CSC model into account makes some difference in understanding evolution in cancer cells because it indicates an inequality in the contribution of cancer cells to clonal evolution. The CSC model distinguishes two functionally distinct populations of cells, one with a virtually unlimited proliferative ability (the CSCS), and the other with a limited proliferative ability (the non-CSCs) (Fig. 1b). On short time scales (days to weeks) this functional distinction makes no difference. But on the time scale of the disease (months to years), it makes a major difference as mutations occurring in non-CSCs are washed away from the population through exhaustion of the proliferative ability of these cells and ultimately cell death. Non-CSCs are evolutionary dead-ends and only the mutations occurring in CSCs can persist over time and generate long-standing subclones (i.e. the various populations of cells with the same pool of mutations that constitute a given cancer) (Fig. 2 a-b). The proportion of mutations that will be able to participate in CE over the course of the disease depends both on the frequency of CSCs in the population (Fig. 2c) and the probability of dedifferentiation of non-CSCs (Fig. 2d). This led Greaves to argue that CSCs are the main units of selection in cancers (Greaves and Maley 2012; Greaves 2013, 2015). The question then is: what difference does it make for CE? Germain (2012) offered an answer: CSC restriction limits evolution by natural selection and makes complex adaptations unlikely.

The main aim of Germain (2012) was to assess the role of evolution by natural selection in cancers. To achieve this goal, he used Godfrey-Smith's (2009) famous account of Darwinian populations and applied it to cancers. The importance of Godfrey-Smith's framework for Germain was that it allows a more precise description of different types of Darwinian populations as functions of roughly measurable parameters; the two extreme types of Darwinian populations being described as paradigmatic Darwinian populations and marginal Darwinian populations. Evolution by natural selection can occur in both but plays a much greater role in the former. The general conclusion of Germain is that although cancer cells are Darwinian populations, they are not paradigmatic populations because there are a number of parameters for which they score rather low (see Table 1 in Germain 2012). My interest here is in the impact of CSC restriction on evolution by natural selection, a question that Germain addressed by scoring cancer cell populations with and without taking into account the CSC model. For one parameter, reproductive specialization, taking the CSC model into account makes a large difference in how the cancer cells score, and therefore on how Darwinian they are. 
a. CE model without taking into account CSCs

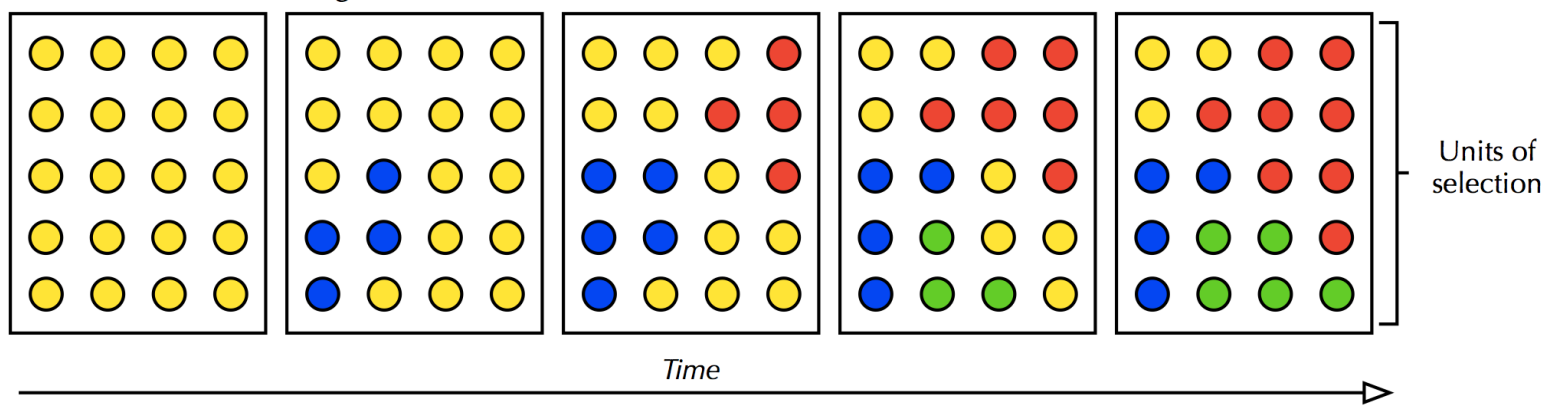

b. CE model with CSC restriction
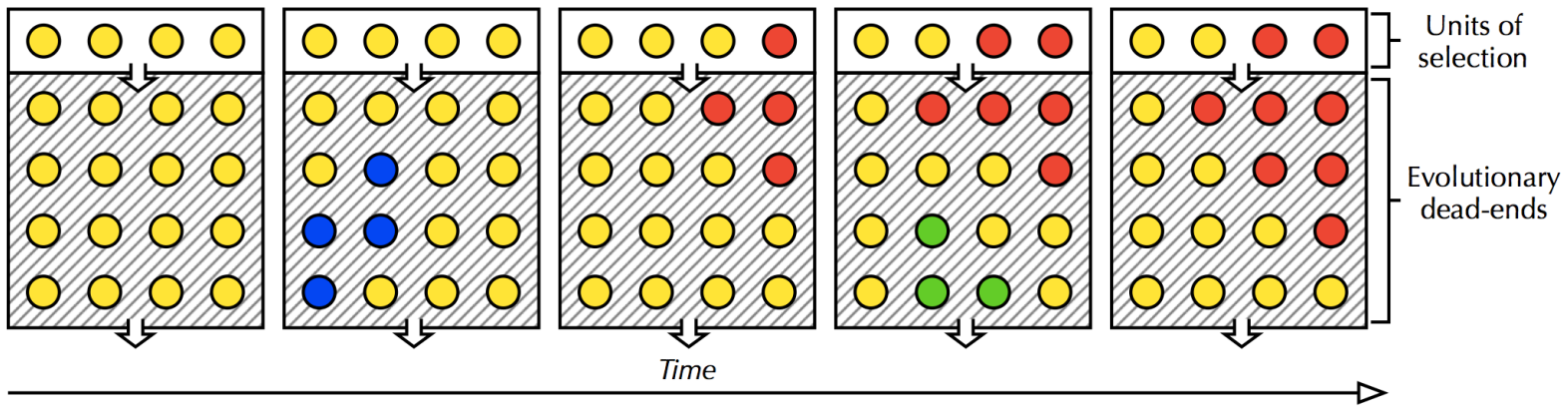

c. The impact of CSC restriction depends on CSC frequency

Low frequency

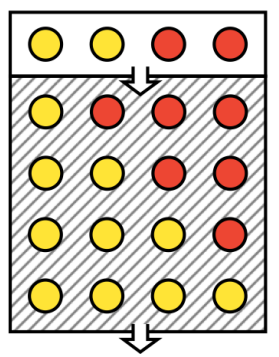

Intermediate frequency

High frequency

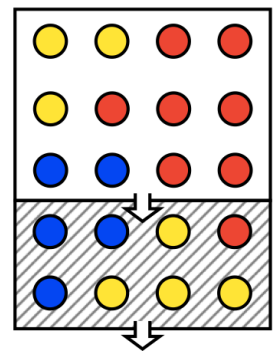

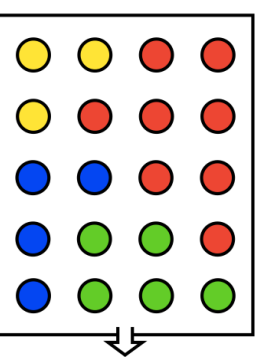

d. The impact of CSC restriction depends on the probability of dedifferentiation of non-CSCs

Low probability

High probability
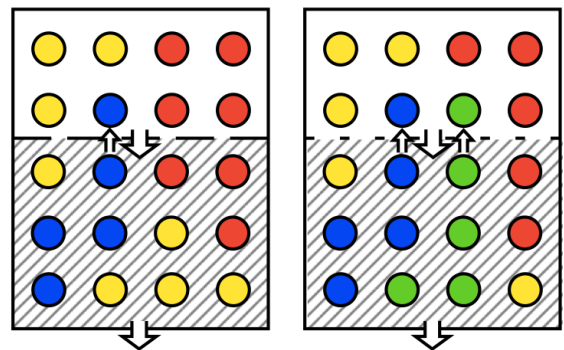

Figure 2 CE with and without taking into account CSC restriction. (a) In the classical CE model, which does not take CSCs into account, all cancer cells are considered to be units of selection. (b) When CSC restriction is taken into account, only the CSCs are considered as meaningful units of selection throughout the course of the disease, because the mutations that occur in non-CSCs are washed away from the population. (c) CSC restriction depends on the frequency of CSCs and can vary from very low to very high, which differentially affects CE. (d) CSC restriction also depends on the probability of dedifferentiation of non-CSCs into CSCs, as mutations occurring in non-CSCs are not washed away if the mutated cells dedifferentiate into CSCs. High or low probability of dedifferentiation will differentially affect CE

Reproductive specialization refers to the germ/soma distinction and related phenomena in which only a sequestered part of the individual can give rise to a new individual through sexual or asexual reproduction (the germ cells), while the rest of the individual cannot (the somatic cells) (see Godfrey-Smith 2009, p. 92). In multicellular organisms, reproductive specialization partially suppresses evolution by natural selection at the cell level by making the somatic cells evolutionary dead-ends (see Godfrey-Smith 2009, p. 101). Germain argued that the coexistence of CSCS and non-CSCs in cancers produces a reproductive specialization comparable to the germ/soma distinction: non-CSCs are unable to grow a tumor like somatic cells are unable to produce a new 
organism, whereas CSCs are able to grow a tumor like germ cells are capable of producing a new organism. Greaves made a similar comparison:

Darwinian models of cancer now need to adopt the concept of cancer stem cells; that small population of self-renewing cells that maintain most of the cancer cell population and, in self-replicating, provide the essential reservoir for further genetic variability and selection, equivalent in evolutionary terms to germ cells (Greaves 2007, 218).

Greaves and Germain agree on the phenomenon: CSC restriction is a mechanism by which a number of mutations are washed away from the population (all those that occur in non-CSCs, dedifferentiation events apart, see Fig. 2). This phenomenon also applies in somatic cells of normal tissues where mutations occurring in non-stem cells are removed from the population due to replication limits of cells (again with the exception of dedifferentiation), and it is now a classic argument that stem cells limit cancer risk (Cairns 1975; Pepper et al. 2007). ${ }^{3}$ Whether reproductive specialization accurately captures this phenomenon is a question that I will address later (section 2.2). The important conclusion here is that the higher the CSC restriction (i.e. the lower the CSC frequency and the probability of non-CSC dedifferentiation), the less cancer cells are paradigmatic Darwinian populations.

From this first conclusion, a second conclusion follows in Godfrey-Smith's framework: the less paradigmatic cancer cell populations are, the less likely complex adaptations are to occur because paradigmatic Darwinian populations are characterized as those in which natural selection can explain the origin of novel traits (complex adaptations) whereas natural selection only changes the distribution of already existing traits (simple adaptations) in marginal Darwinian populations (see Godfrey-Smith 2009, chapter 3). Complex adaptations rely on cumulative evolution:

It can seem odd to say that selection, which has to do with sorting things that already exist, can somehow bring new things into existence. But natural selection can reshape a population in a way that makes a given variant more likely to be produced via the immediate sources of variation than it otherwise would be. Selection does this by making intermediate stages on the road to some new characteristic common rather than rare, thus increasing the number of ways in which a given mutational event (or similar) will suffice to produce the characteristic in question. Some kinds of novelty can be produced easily by an evolutionary process without this role for selection, but other kinds-complex and adapted structurescannot (Godfrey-Smith 2009, 43).

CSC restriction impedes complex adaptations by limiting cumulative evolution in two ways (Germain 2012). First, CSC restriction limits the size of the cancer cell population in which evolutionary changes can accumulate (Fig. 2). Any complex adaptations in non-CSCs are very unlikely to occur given that non-CSCs can only proliferate for short periods of time, and thus mutations would be quickly lost. Second, CSCs can be quiescent (i.e. non dividing for long periods of time), which limits the possibility of cumulative evolution in the CSC population itself.

\footnotetext{
${ }^{3}$ Tomasetti and Vogestein (2015) argued that "variation in cancer risk among tissues can be explained by the number of stem cell division," which started a massive debate on cancer etiology and on the causal contribution attributable to environment, heredity, and stem cell replication (see the latest response of Tomasetti et al. 2017).
} 
Germain convincingly argued that the CSC model, and more precisely what I call the level of CSC restriction, plays an important role in what we can expect from evolution by natural selection in cancers. In particular, the higher the CSC restriction, the less likely complex adaptations are to appear (Figure 3). ${ }^{4}$ More generally, CSC restriction is a way to wash out a great amount of the mutations that occur in a given cell population. This not only limits evolution by natural selection but limits CE in general.

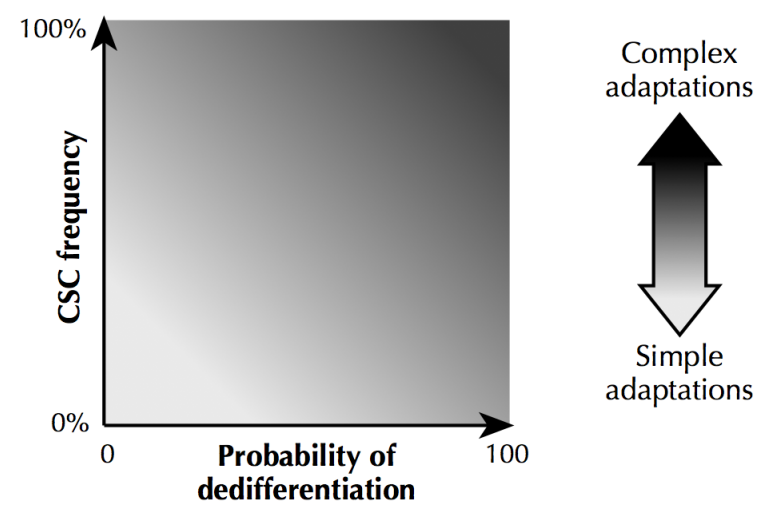

Figure 3 Adaptations depend on CSC restriction. The lower the CSC frequency and the lower the probability of non-CSC dedifferentiation, the more unlikely complex adaptations are to occur. The black and white gradient indicates directions: the lower the CSC frequency and the probability of dedifferentiation of non-CSCs into CSCs, the less likely complex adaptations are. The exact shape of the gradient remains to be empirically evaluated. Notice also that some values are biologically absurd or equivalent. For example, having a CSC frequency of $100 \%$ and a probability of dedifferentiation of 1 is a bit absurd, because if there are only CSCs then there are no nonCSCs to dedifferentiate. Similarly, if the probability of dedifferentiation is 1 then CSC frequency cannot be $0 \%$

\subsection{CSC restriction favors drift}

Germain's investigation focused on evolution by natural selection. As CE is not restricted to evolution by natural selection, I would like to continue his investigation at a larger scale. This section focuses on drift. The CE model was originally framed with the idea that cancer is a Darwinian process of gradual evolution by natural selection. ${ }^{5}$ Yet, since Germain's article was published, evidence for neutral evolution has been reported in hepatocellular carcinoma (Ling et al. 2015) and colorectal cancers (Sottoriva et al. 2015; Sottoriva et al. 2017). Applying a neutral evolution mathematical model to the sequencing data of 904 cancers from 14 types, Williams et al. (2016) found that the neutral model fitted with high precision 323 of them (more than a third), suggesting that neutral evolution could affect many more cancers than anticipated. In these cancers, they argue, most of the mutations responsible for the cancer expansion were already present in the first malignant cell and the following evolution was neutral, a phenomenon sometime referred to as a "Big Bang" model of cancer progression (Sottoriva et al.

\footnotetext{
${ }^{4}$ I focus on mutations because they are the usual traits that are used to reconstruct and study the evolution of cancer cell populations, but the argument would apply for any heritable trait.

${ }^{5}$ Here I focus on the assumption that CE is a process of evolution by natural selection because, to the best of my knowledge, no evidence suggests that CSC restriction can favor or limit punctuated or gradual evolution. But the assumption that $\mathrm{CE}$ is a gradual process has been challenged by the observation of several complex mutational events that induce multiple mutations in a burst (e.g. Stephens et al. 2011; Rausch et al. 2012; Nik-Zainal et al. 2012a; Baca et al. 2013). The extent and limits of the analogy with Eldredge and Gould's punctuated evolution is discussed in Gao et al. (2016). Markowetz (2016) suggested that saltationist theories such as the hopeful monsters theory of Richard Goldschmidt might be more relevant in cancers.
} 
2015; Amaro et al. 2016). ${ }^{6}$ Another pan-analysis also highlighted that negative selection, which is predominant in the germ line, is nearly absent in cancer and somatic evolution (Martincorena et al. 2017). Does CSC restriction impact whether cancers follow a pattern of neutral evolution? A model developed by Sottoriva et al. (2010) suggests as much. In this model, Sottoriva and colleagues observed that CE was impacted in two ways when they factored in the CSC model (i.e. by distinguishing two functionally distinct populations of cancer cells, CSCs and non-CSCs). First, as already discussed in section 1.1, CSCs repress CE by making the acquisition of mutations slower. Second, they observed a different clonal expansion process. When the CSC model is not taken into account, a small number of the occurring mutations were selected for and invaded large portions of the cancer cell population. In contrast, the implementation of the CSC model resulted in the persistence of a large proportion of mutations that remained at a low frequency in the population, consistent with neutral evolution. They concluded "that the intrinsic properties of the CSC model might propel an alternative process to natural selection, referred to as genetic drift" (Sottoriva et al. 2010, 52), and attributed the occurrence of drift to sampling errors that are much more frequent in small populations-the CSC model stimulates sampling error mechanisms by limiting the proportion of cells that can acquire and maintain mutations.

The conclusion of Sottoriva et al. (2010) that CSC restriction favors drift raises the question of whether there is higher CSC restriction in the cancers that fitted the neutral model in Williams et al. (2016). To answer this question, one would have to align the analysis of neutral evolution from whole genome sequencing data with scorings of CSC frequency and probability of dedifferentiation in various cancer types and also in cancers at various stages of progression. This would show how a change in CSC restriction can impact the processes of CE (Fig. 4).

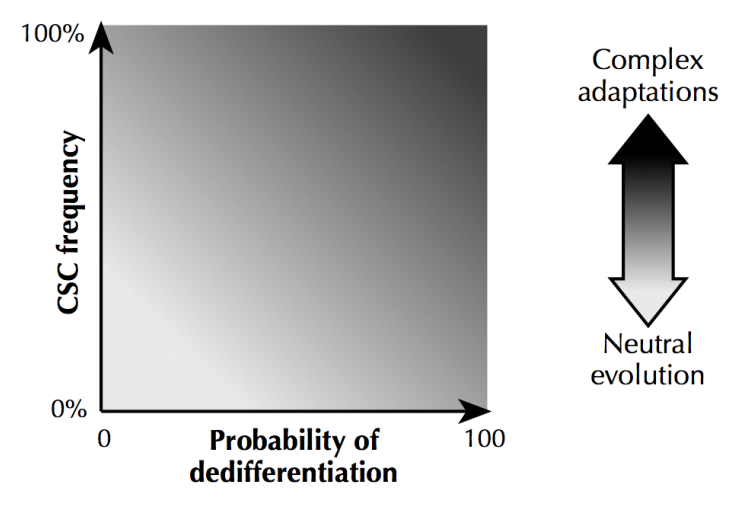

Figure 4 CSC restriction and drift. The axes in this schematic diagram are the same as the previous diagram (Fig. 3). Lower CSC frequency and probability of dedifferentiation not only make complex adaptations very unlikely, they also favor drift. Empirical comparison between processes of evolution and level of CSC restriction are required to characterize the $C E$ processes associated with each of the points of this two-dimensional space

\subsection{CSC restriction favors linear evolution}

${ }^{6}$ Whether a first stage of evolution by natural selection precedes neutral evolution is a matter of debate (Martincorena et al. 2015; Simons 2016). 
The CE model was first represented as a linear model of evolution wherein mutations accumulate sequentially, with each new clone outcompeting the previous one, so that subclones accumulating new mutations proceed one after another (Fig. 5a). In this pattern of CE, intratumoral heterogeneity (ITH) is diachronic rather than synchronic, such that cells accumulate mutations through time but the mutations appear homogenous throughout the cell population at any given time (i.e. at T1 all cells have mutation 1 , at T2 all cells have mutations 1 and 2, etc.)(e.g. Hou et al. 2012). In most cases of linear evolution though, selective sweeps appear incomplete and former subclones persist, but do not give rise to new subclones (Fig. 5b) (e.g. Merlevede et al. 2016). When they do give rise to new subclones, the result is a branched pattern of evolution: mutations randomly accumulate in cells of various subclones continuously leading to the appearance of new subclones (Fig. 5c) (e.g. Yachida et al. 2016), which is the most common case (Andor et al. 2016). In this section I develop the hypothesis that CSC restriction might also impact patterns of evolution, and more precisely that a higher level of CSC restriction might favor linear evolution.

Linear
Homogeneous

Figure 5 Three patterns of CE. (a) Linear homogeneous CE is characterized by the sequential accumulation of mutations with each new clone outcompeting the previous so that subclones accumulating new mutations succeed one another. (b) When selective sweeps are incomplete and former subclones persist without giving rise to new subclones, the CS is linear heterogeneous. (c) When former subclones accumulate mutations and give rise to new subclones, CE is branched: mutations randomly accumulate in cells of various subclones continuously leading to the appearance of new subclones

With similar cell division and mutation rates in a fixed period of time, a smaller population will accumulate fewer mutations than a bigger population. This is true by definition if mutations occur during division. When a mutation does occur, all things being equal, it should also have a higher probability of invading the population, as there are fewer cells to outcompete. There is thus an inverse correlation between the probability of occurrence of new mutations and the probability of propagation of the mutations. If mutations are less frequent and propagate more easily in a smaller population, then there is a higher chance that the second mutation occurs on top of the 
first mutation (because the first mutation is present in a larger fraction of the population at the time of occurrence of the second mutation). If the second mutation does occur on top of the first, and the third on top of the second, then we have linear evolution. In contrast, in a very large population, mutations propagate less easily and occur more frequently, leaving less time for the previous mutation to propagate, and reducing the chance that a new mutation occurs in a cell bearing the previous one. If the new mutation occurs in a cell that does not have the previous mutation, then we have branching evolution. Thus, ceteris paribus, smaller populations are more likely to undergo linear evolution and larger populations are more likely to undergo branching evolution. As only CSCS can participate in CE over the course of the disease, it is actually the CSC restriction that matters for patterns of CE. Higher CSC restriction should thus favor linear CE, whereas a loss of CSC restriction by an increased CSC frequency and/or an increased probability of dedifferentiation should increase the probability of branching evolution (Fig 6a).

The relationship between CSC restriction and patterns of CE has not yet been studied, but there is some indirect evidence in favor of this hypothesis. First, to the best of my knowledge, all cases of linear evolution that have been reported are in blood cancers (supplementary table 1, grey lines), which are characterized by a low CSC frequency and no dedifferentiation, thus a high CSC restriction. The only exception comes from Shain et al. (2015) who reported linear evolution in the premalignant stages of melanocytic neoplasms, suggesting that in solid tumors, the initial premalignant stage could follow a linear pattern of evolution before exploding into a branched pattern of evolution at initiation of the malignant stage (which would be in line with the fact that cancers start from the mutation of a single cell and thus with a small population of CSCs) (Fig 6b). Cancers in which non-CSCs can dedifferentiate and CSC frequency is higher, such as breast cancers, colon cancers, advanced melanoma, brain cancers, osteosarcoma, and pancreatic cancers, follow branched patterns of evolution (supplementary table 1). The second kind of evidence comes from patterns of accumulation of mutations in normal tissues (i.e. without any overt cancer being diagnosed). Presence of mutations usually associated with cancers without overt malignancies have been well documented in two tissues: skin of the eyelid and blood. Stem cell restriction is much lower in the former tissue than in the latter (higher stem cell frequency and dedifferentiation have been reported in skin epithelium). Interestingly, the patterns of mutations in these tissues are very different. In the eyelid, Martincorena et al. (2015) observed a high rate of mutations giving rise to numerous but very small clones (i.e. clones with small numbers of cells). In contrast, in the blood very few mutations have been observed, but these give rise to bigger clones (Genovese et al. 2014; Jaiswal et al. 2014; Xie et al. 2014).

To conclude this first section, CSC restriction limits evolution by natural selection, and more generally CE, prevents complex adaptations, and favors drift and linear evolution in a manner that depends on CSC frequency and the probability of dedifferentiation (Fig 3, 4 and 6). By ignoring the CSC model or by considering it as an independent model of cancer, the CE model misses these impacts of CSC restriction on CE characteristics. 
a. Patterns of CE and CSC restriction

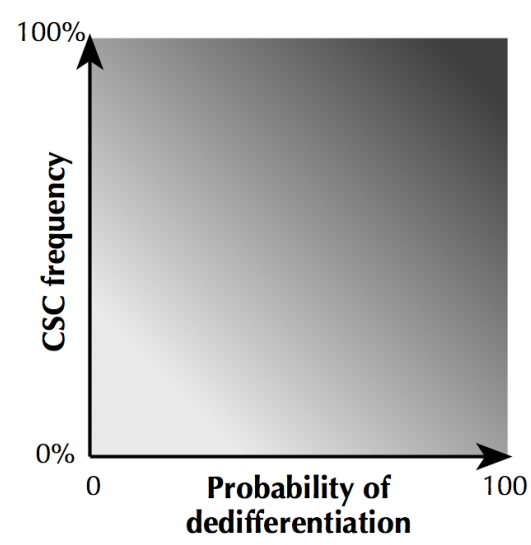

b. Disease progression

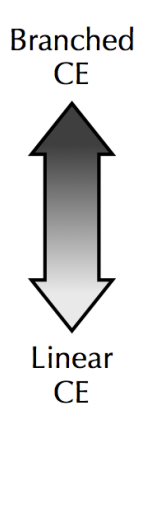

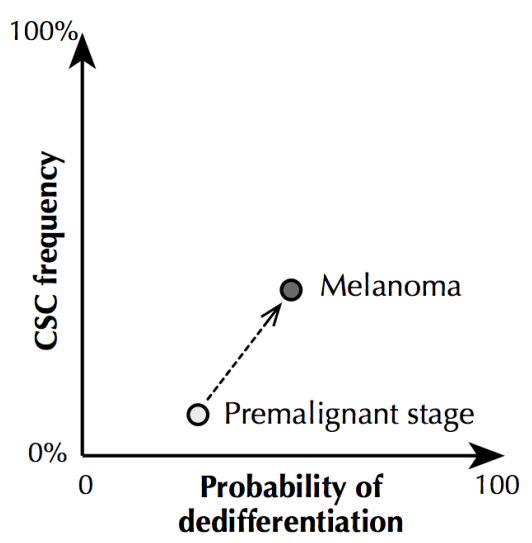

Figure 6 Patterns of evolution and CSC restriction. (a) Lower CSC frequency and lower probability of dedifferentiation favors linear evolution. (b) During disease progression, cell populations might move in this twodimensional space. A loss of CSC restriction through an increase in CSC frequency and/or increase in probability of dedifferentiation might favor a transition from a linear CE to a branched CE

\section{Traditional parameters of evolution do not accurately describe the consequences of CSC restriction for CE}

The first section showed that the CE model should take into account the CSC model, and more precisely CSC restriction, because it can affect both the patterns and the processes of CE. This distinction between CSCS and non-CSCs and its impact on CE resemble some phenomena already well known and described in evolutionary biology. Scientists and philosophers have discussed the resemblance with various parameters of evolution such as heredity (Greaves 2013), reproductive specialization (Germain 2012; Greaves 2013), and effective population size (e.g. Merlo et al. 2006; Pepper et al. 2009; Sottoriva et al. 2010; Lipinski et al. 2016; Lean and Plutynski 2016). This section presents and discusses these comparisons as well as additional ones (bottleneck and dependence of fitness on intrinsic properties). I argue that none of these parameters are entirely satisfactory to describe the impact of CSC restriction on CE and show that the difficulties come from the fact that CSC restriction depends on two factors: the CSC frequency and the probability of dedifferentiation of non-CSCs into CSCs.

\subsection{CSC restriction and heredity}

Referring to Lewontin's classical minimal requirement for evolution by natural selection, Greaves has argued that it is the lack of heredity that suppresses CE in non-CSCs, so that only CSCs are genuine units of selection in cancers (e.g. Greaves 2013, 104). This claim relies on a misunderstanding of the parameter of heredity. Heredity, as a parameter of evolution, refers to the fidelity of heredity at each generation. So what is scored in heredity is the "similarity between parent and offspring, due to causal role of the parents" (Godfrey-Smith 2009, 39). In cancers, the fidelity of heredity largely depends on the quantity of mutations, which is highly variable. For example, comparing 30 cancer types, Alexandrov et al. (2013) reported a mutational load ranging from about 0.001/Mb to more than $400 / \mathrm{Mb}$. Whereas melanoma can present thousands of mutations, and thus a very low fidelity of heredity during cell division, very few mutations, if any, were reported in ependymomas (a pediatric tumor of the central nervous system) indicating a high fidelity of heredity (Mack et al. 2014). These differences depend on several factors such as genetic instability, or exposure to mutagenic agents. For example, the fidelity of heredity 
is far lower in lung cancers of smokers than of non-smokers (Alexandrov et al. 2016). CSC restriction plays little role in fidelity of heredity. What Greaves meant by lack of heredity was that non-CSCs can only give rise to a limited number of generations of cells and that only CSCs can reproduce in the long-term, a fact we all agree on but struggle to depict appropriately.

\subsection{CSC restriction, reproductive specialization and bottleneck}

Referring to Godfrey-Smith's now famous parameters of evolution, Germain (2012) claimed that CSC restriction changes the degree of reproductive specialization (see section 1.1). I agree that there is an analogy between germ line sequestration in multicellular organisms and CSC restriction in cancers. There is, in both cases, a division of labor that has massive impacts on the long-term fitness of the cells. But this analogy has several limitations. Reproductive specialization refers to the germ/soma distinction, or equivalent phenomena. It relies on three conditions. There is a reproductive specialization when: (1) there is reproduction; (2) only a part of the cell population is involved in this reproduction; and (3) these cells are the germ cells, which are distinct from somatic cells, that is, they belong to a separated cell line.

Whether there is reproduction within cancers is a debatable matter. Metastases could be a candidate (see Lean and Plutynski 2016; Germain and Laplane 2017), but my argument is that CSC restriction impacts patterns and processes of $\mathrm{CE}$ at the cellular level, regardless of the formation of metastases. One could then argue that cell lineages that make up each tumor are individuals that reproduce. In a sense, each CSC gives rise to a lineage containing a body of non-CSCs. When a CSC self-renews and produces a new CSC that gives rise to a new lineage, then do we have a case of reproduction? Whether each lineage can count as an individual seems debatable to me given the absence of integration. ${ }^{7}$ But, if we agree that there is a genuine case of reproduction here, then we also meet the second criteria for reproductive specialization, namely that only a part of the cells constituting the individual are involved in the reproduction, here the CSCs. However, we then face a limitation in the analogy with regard to the third criterion: CSCs and non-CSCs are not two separated cell lines like somatic and germ cells are. While germ line cells are sequestered, CSCs (as well as normal stem cells) are not, as the CSCs give rise to the non-CSCs. For CE, this might be considered as a negligible problem when the probability of dedifferentiation of non-CSCs into CSCs is null. But it becomes a major difference as soon as non-CSCs can become CSCs. In this case the distinction between germ line and soma can no longer apply. If there is reproduction, then it is of a very different kind, one that would involve a bottleneck.

A bottleneck is when reproduction occurs from only a small part of the parental organism (see Godfrey-Smith 2009, chapter 5). At first sight, bottleneck and reproductive specialization might seem very similar, as both are processes that wash away some mutations, which is exactly what the CSC vs. non-CSC distinction does. However, they differ in their consequences for evolution: reproductive specialization prevents any mutation occurring in the somatic cells/non-CSCs from being transmitted to the next generation, whereas a bottleneck does not as virtually any cell can be transmitted to the next generation. Thus, in cases where non-CSCs can dedifferentiate

\footnotetext{
${ }^{7}$ There are some feedback loops between mature cells and CSCs (e.g. Reynaud et al. 2011), but those are not restricted to their own lineages and in some cases, like blood cancers, the cells from different lineages are all mixed and moving around.
} 
into CSCs, the question becomes whether CSC restriction (CSC frequency and probability of dedifferentiation) equates to the degree of bottleneckishness. If each CSC lineage counts as an individual, then the degree of bottleneckishness is always the same: each individual starts with only one CSC, whatever the probability is of dedifferentiation. If the degree of bottleneckishness is always the same while the CSC restriction can vary, then it cannot describe the degree of CSC restriction.

Hence, the reproductive specialization parameter could only capture the impact of CSC restriction on CE in cases where the probability of dedifferentiation is null, with the limitations in the analogy that CSCs and non-CSCs are not separate lineages and provided that lineages do reproduce. One could then argue that the frequency of CSCs equates to the degree of reproductive specialization. But CSC restriction also depends on the probability of dedifferentiation, and when non-CSCs can dedifferentiate into CSCs, then there is no reproductive specialization, but rather a bottleneck. However, as the degree of bottleneckishness is stable (one cell, the CSC), it is unlikely to do a better job of capturing the impact of CSC restriction on CE, which changes with the probability of dedifferentiation and CSC frequency. ${ }^{8}$

\subsection{CSC restriction and dependence of fitness on intrinsic properties}

Besides being a case of reproductive specialization, Godfrey-Smith (2009) also describes germ line sequestration as a case of low dependence of fitness on intrinsic properties, which quantifies how much reproductive success depends on intrinsic properties (e.g. the genetic content of the cells) versus extrinsic properties (e.g. the location in a particular environment). Low dependence of fitness on intrinsic properties represses evolution by natural selection, as in the case of germ line sequestration, because the difference in the long-term fitness of the germ line cells compared to somatic cells is not due to intrinsic properties (that can be positively selected for) but to the fact that the cells ended up being part of one or another group of cells during development. If reproductive specialization does not exactly capture CSC restriction, can dependence of fitness on intrinsic properties do a better job? The question here is whether the difference in fitness between CSCs and non-CSCs is due to context or intrinsic properties. In other words, is CSC identity intrinsic or extrinsic?

If non-CSCs can dedifferentiate into CSCs, it means that under certain circumstances in some cancers, stemness can be acquired. But the ability or not to dedifferentiate is not sufficient to answer our question, as the microenvironment can play varying roles in both cases. In previous work, I have shown that two questions must be distinguished to understand the identity of stem cells, including CSCs: (1) can non-stem cells/non-CSCs acquire stemness and dedifferentiate into stem cells/CSCs? (2) Is stemness acquisition and/or maintenance dependent upon the microenvironment? Depending on the answers to these questions, which are specific to tissues and cancers, four different identities can be distinguished for the property of stemness (Laplane 2016):

1. Categorical: stemness is purely intrinsic.

\footnotetext{
${ }^{8}$ I am very grateful to both anonymous reviewers for the thorough discussion that has lead to this version of the
} argument. 
2. Dispositional: stemness is an intrinsic property whose expression depends on extrinsic stimuli from the microenvironment.

3. Relational: stemness is an extrinsic property induced and controlled by the microenvironment.

4. Systemic: stemness is an extrinsic property controlled at the cell population level.

In the last three cases, the fitness of the CSCs depends, to various degrees, on context (which can include the tumor-microenvironment, clone-clone interactions, and feedback loops between mature cells and CSCs). In cases where stemness is a relational property, being a CSC depends on the location of the cancer cells. Dependence of fitness on intrinsic properties, in this instance, is very low. It is higher in cases where stemness is a dispositional property because being a CSC depends on intrinsic properties, although it is still limited by the fact that the reproductive success of each CSC is regulated by the microenvironment. In cases where stemness is a systemic property, being a CSC depends on various factors including intrinsic properties such as stochastic gene expression and extrinsic properties such as feedback loops and other collective processes; here, dependence of fitness on intrinsic properties hangs upon the relative contribution of intrinsic and extrinsic factors in stemness. Finally, dependence of fitness on intrinsic properties is high in cases where stemness is a categorical property because the difference in fitness in the long term is mainly due to intrinsic properties. Thus, the identity of CSCs can provide interesting insights for $\mathrm{CE}$, for example, cancers in which stemness is a categorical property should be more likely to evolve by natural selection than cancers in which stemness is a relational property. But, the degree of dependence of fitness on intrinsic properties does not correlate with the degree of CSC restriction.

\subsection{CSC restriction and effective population size}

Some biologists and philosophers have suggested that CSC restriction simply limits the effective population size, as opposed to the census population size (e.g. Merlo et al. 2006; Pepper et al. 2009; Sottoriva et al. 2010; Lipinski et al. 2016; Lean and Plutynski 2016).

In fact, the question of whether the entire neoplasm or a minority of neoplastic cells is capable of self-renewal is, at least in part, a question merely of the effective population size of the evolving cells in a neoplasm (Pepper et al. 2009,66).

The claim here is that the effective population size is the size of the CSC population. The lower the CSC frequency is, the greater the difference is between the effective population size and the census population size, and the smaller the effective population size is. Reducing the effective population size impacts evolutionary processes: smaller populations are considered more prone to drift, as sampling error can more easily lead to the expansion of clones with no clear survival benefit (see section 1.2). ${ }^{9}$ More generally, the arguments that CSC restriction represses CE, favors drift, prevents complex adaptations, and favors linear evolution, could be reduced to a question of effective population size, if the probability of dedifferentiation is null. But if non-CSCs can dedifferentiate into CSCs at any time, then the actual CSC frequency poorly represents the cells that can

\footnotetext{
${ }^{9}$ The idea that drift dominates selection in small populations has been debated, see for example the recent
} debate between Clatterbuck, Sober and Lewontin (2013) and Brandon and Fleming (2014). 
meaningfully contribute to $\mathrm{CE}$, and thus also poorly represents the effective population size. The probability of dedifferentiation of non-CSCs into CSCs is then once more a major factor for CE.

Taken together, these difficulties highlight that CSC restriction is not easily captured by any classical parameter of evolution, in particular because of the difference between CSC frequency and dedifferentiation, as well as the four different possible identities of CSCS (see section 2.3). To account for the impact of CSC restriction on CE, one needs to measure both the frequency of CSCs and the probability of dedifferentiation of non-CSCs.

\section{Biomedical relevance of the impact of CSC restriction on CE}

The first section showed that the degree of CSC restriction could favor or impede various processes and patterns of evolution, and in particular that it can favor linear or branched evolution. Scientists regularly investigate whether CE is linear or branched but give limited attention to the difference it makes. Why should we care about the impact of CSC restriction on linear or branched evolution? In this section, I argue that the relationship between CSC restriction and patterns of CE is worth exploring for the biomedical sciences, first, because the patterns of CE can inform us about CSC restriction, and in particular about the probability of dedifferentiation that is currently difficult to measure; second, because it offers original opportunities for new therapeutic strategies.

\subsection{From CE patterns to CSC restriction}

CE patterns are mainly used to infer either the relative fitness of each subclone in a given environment or their age. In cases of evolution by natural selection the bigger subclones are the ones with the greatest reproductive success in that environment. In cases of neutral evolution, the bigger subclones are the oldest. In addition to these inferences, I have argued in this paper that the pattern of CE-linear or branched-is, at least in part, a function of CSC restriction. If I am right, then reconstruction of the CE could be informative about CSC restriction. This could be valuable in cancers in which one of the two parameters of CSC restriction-CSC frequency and probability of dedifferentiation - is unknown. For example, in a set of cancers of a given type (e.g. melanomas), if we can measure the CSC frequency and reconstruct the CE tree, we may be able to infer if dedifferentiation occurred or not and, if it occurred, whether it is a marginal or frequent process (i.e. whether the probability of dedifferentiation is high or low). This is far from trivial given that the current technologies allow far better approximations of CE patterns and of CSC frequencies than of the probability of dedifferentiation.

\subsection{From CSC restriction to CE patterns: therapeutic consequences}

What difference does it make if CE is linear or branched? Is one or the other better for the patients? The immediate relevance of distinguishing CE patterns is that the coexistence of multiple subclones is a cause of therapy resistance. The more subclones there are, the greater the risk of therapy resistance. If my hypothesis that a higher CSC restriction should favor a more linear and simpler pattern of CE is right, then patients would benefit from any complementary therapies that can increase CSC restriction. Opportunities to control CSC restriction are emerging. The most recent examples are Cimmino et al. (2017) and Agathocleous et al. (2017), who showed that vitamin $\mathrm{C}$ regulates the frequency of normal or malignant hematopoietic stem cells. 
Conversely, any therapy that would decrease CSC restriction should be avoided. Hypomethylating agents (HMAs) are used as first line treatment in chronic myelomonocytic leukemia (CMML), myelodysplastic syndromes (MDS), and acute myeloid leukemia (AML) of the elderly, when patients are not eligible for stem cell transplantation. But HMAs have also been used in a very different context, unrelated to cancer, to induce cell plasticity and dedifferentiation (e.g. Mikkelsen et al. 2008; Chandrakanthan et al. 2016). Some studies also suggest that HMAs can decrease stem cell restriction in the normal hematopoietic system, either by increasing their frequency or by inducing dedifferentiation (Suzuki et al. 2004; Chung et al. 2009). If they also decrease CSC restriction in the patients (a question I am currently experimentally investigating in my lab), then the prediction of this study is that HMA treatment must favor more CE, more adaptability, and more complex patterns of evolution. This could help to explain the transiency of patients' responses to HMAs and the frequent progression of CMML and MDS into AML. If progression under treatment were due to the loss of CSC restriction, then one way to maintain the benefit of the drug without its counterproductive effects would be to couple it with a drug increasing CSC restriction. Many drugs (none targeting CSCs) are currently tested in combination with HMAs without yet leading to improvement in overall survival (Ball et al. 2017). In this current clinical context, my work suggests that drugs acting on CSC restriction are worth investigating too.

\section{Conclusion and perspectives}

While the inclusion of CE into the CSC model has been fairly well addressed in the literature (Kreso and Dick 2014; Nassar and Blanpain 2016), showing in particular that CSCs are a heterogeneous target for anti-CSC therapies, the impact of the CSC model on CE has been poorly explored. This article shows that CSC restriction, which depends on CSC frequency and the probability of non-CSC dedifferentiation, impacts CE in several ways. The higher the CSC restriction, the more it can:

- $\quad$ limit CE;

- limit evolution by natural selection;

- $\quad$ prevent complex adaptations;

- favor drift;

- favor linear evolution.

A loss of CSC restriction (higher CSC frequency and/or higher probability of dedifferentiation) during disease progression will thus favor more adaptability, making the inclusion of CSC restriction into the CE model highly relevant for biomedical research.

Incorporating CSC restriction into the CE model faces several difficulties, opening perspectives for further philosophical and biological research. First, within current experimental limitations, it is difficult to properly quantify the number of CSCs and the probability of dedifferentiation within a cancer at any given time. Thus CSC restriction can only be roughly estimated. Current improvements in lineage tracing technologies will provide great help. Estimation of the probability of dedifferentiation of non-CSCs into CSCs is also becoming an urgent need that remains too poorly addressed. 
Second, recent work has shown that under homeostatic conditions, progenitor cells (the first daughter cells of stem cells), can support the production of all the blood cells for much longer than initially thought (Sun et al. 2014; Busch et al. 2015). This raises a new question for cancers: are CSCs the only meaningful units of selection in CE? Depending on the duration of the disease, progenitor cells might also be taken into account as part of CSC restriction, which would change the measure of CSC restriction.

Third, the analysis presented in this article is made ceteris paribus. My point is that every other parameter being equal, a difference in CSC restriction will affect CE. A further question would be whether other parameters, like the rate of mutation and of proliferation, as well as the role of the microenvironment and more generally the physical constraints of the tissues outweigh some of the impact of CSCS on the patterns and processes of CE.

Finally, this article focuses on the impact of CSC restriction on CE. If correct, then the next question is how can we act on CSC restriction in order to limit CE and disease progression? To answer this question requires investigating the two factors of CSC restriction: CSC frequency and non-CSC dedifferentiation. What causes an increase in the frequency of CSCs within a given cancer, and how can this increase in the number of CSCs be avoided or reduced? What induces dedifferentiation of non-CSCs into CSCs and how can we prevent it? These questions require a thorough investigation of the role of the microenvironment and of population-level processes. The microenvironment can play a decisive role in the proliferation of CSCs and their ability to generate a clone (Arranz et al. 2014; Dong et al. 2016). Mature cancer cells (non-CSCs) can also play an important role in the proliferation of the CSCs through the cytokines they secrete (Reynaud et al. 2011; Welner et al. 2015), raising the question of interactions between subpopulations, and more generally indicating the need to investigate multilevel selection processes (Sprouffske et al. 2013; Lean and Plutynski 2016).

\section{References}

Adams JM, Strasser A (2008) Is tumor growth sustained by rare cancer stem cells or dominant clones? Cancer Res 68:4018-4021 . doi: 68/11/4018 [pii]10.1158/0008-5472.CAN-07-6334

Agathocleous M, Meacham CE, Burgess RJ, et al (2017) Ascorbate regulates haematopoietic stem cell function and leukaemogenesis. Nature 549:476-481 . doi: 10.1038/nature23876

Alexandrov LB, Ju YS, Haase K, et al (2016) Mutational signatures associated with tobacco smoking in human cancer. Science 354:618-622 . doi: 10.1126/science.aag0299

Alexandrov LB, Nik-Zainal S, Wedge DC, et al (2013) Signatures of mutational processes in human cancer. Nature 500:415-21 . doi: 10.1038/nature12477

Amaro A, Chiara S, Pfeffer U (2016) Molecular evolution of colorectal cancer: from multistep carcinogenesis to the big bang. Cancer Metastasis Rev 35:63-74 . doi: 10.1007/s10555-016-9606-4

Anderson K, Lutz C, van Delft FW, et al (2011) Genetic variegation of clonal architecture and propagating cells in leukaemia. Nature 469:356-361 . doi: nature09650 [pii] 10.1038/nature09650

Andor N, Graham TA, Jansen M, et al (2016) Pan-cancer analysis of the extent and consequences of intratumor heterogeneity. Nat Med 22:105-113 . doi: 10.1038/nm.3984

Arranz L, Sánchez-Aguilera A, Martín-Pérez D, et al (2014) Neuropathy of haematopoietic stem cell niche is 
essential for myeloproliferative neoplasms. Nature 512:78-81 . doi: 10.1038/nature13383

Baca SC, Prandi D, Lawrence MS, et al (2013) Punctuated evolution of prostate cancer genomes. Cell 153:66677 . doi: 10.1016/j.cell.2013.03.021

Ball B, Zeidan A, Gore SD, Prebet T (2017) Hypomethylating agent combination strategies in myelodysplastic syndromes: hopes and shortcomings. Leuk. Lymphoma 58:1022-1036

Bapat SA (2007) Evolution of cancer stem cells. Semin Cancer Biol 17:204-213 . doi: S1044-579X(06)00039-3 [pii]10.1016/j.semcancer.2006.05.001

Batlle E, Clevers H (2017) Cancer stem cells revisited. Nat Med 23:1124-1134 . doi: 10.1038/nm.4409

Bolli N, Avet-Loiseau H, Wedge DC, et al (2014) Heterogeneity of genomic evolution and mutational profiles in multiple myeloma. Nat Commun 5: . doi: 10.1038/ncomms3997

Bonnet D, Dick JE (1997) Human acute myeloid leukemia is organized as a hierarchy that originates from a primitive hematopoietic cell. Nat Med 3:730-737 . doi: 10.1038/nm0797-730

Brabletz T, Jung A, Spaderna S, et al (2005) Opinion: migrating cancer stem cells - an integrated concept of malignant tumour progression. Nat Rev Cancer 5:744-749 . doi: nrc1694 [pii]10.1038/nrc1694

Brandon R, Fleming L (2014) Drift sometimes dominates selection, and vice versa: a reply to Clatterbuck, Sober and Lewontin. Biol Philos 29:577-585 . doi: 10.1007/s10539-014-9437-z

Brocks D, Assenov Y, Minner S, et al (2014) Intratumor DNA methylation heterogeneity reflects clonal evolution in aggressive prostate cancer. Cell Rep 8:798-806 . doi: 10.1016/j.celrep.2014.06.053

Busch K, Klapproth K, Barile M, et al (2015) Fundamental properties of unperturbed haematopoiesis from stem cells in vivo. Nature 518:542-546 . doi: 10.1038/nature14242

Cairns J (1975) Mutation selection and the natural history of cancer. Nature 255:197-200

Campbell LL, Polyak K (2007) Breast tumor heterogeneity: cancer stem cells or clonal evolution? Cell Cycle 6:2332-2338 . doi: 4914 [pii]

Cao W, Wu W, Yan M, et al (2015) Multiple region whole-exome sequencing reveals dramatically evolving intratumor genomic heterogeneity in esophageal squamous cell carcinoma. Oncogenesis 4:e175 . doi: 10.1038/oncsis.2015.34

Caye A, Strullu M, Guidez F, et al (2015) Juvenile myelomonocytic leukemia displays mutations in components of the RAS pathway and the PRC2 network. Nat Genet 47:1334-1340 . doi: 10.1038/ng.3420

Chaffer CL, Marjanovic ND, Lee T, et al (2013) Poised Chromatin at the ZEB1 Promoter Enables Breast Cancer Cell Plasticity and Enhances Tumorigenicity. Cell 154:61-74 . doi: http://dx.doi.org/10.1016/j.cell.2013.06.005

Chandrakanthan V, Yeola A, Kwan JC, et al (2016) PDGF-AB and 5-Azacytidine induce conversion of somatic cells into tissue-regenerative multipotent stem cells. Proc Natl Acad Sci U S A 113:E2306-15 . doi: 10.1073/pnas.1518244113

Chesnais V, Renneville A, Toma A, et al (2016) Effect of lenalidomide treatment on clonal architecture of myelodysplastic syndromes without 5q deletion. Blood 127:749-60 . doi: 10.1182/blood-2015-04-640128

Chung YS, Kim HJ, Kim T-M, et al (2009) Undifferentiated hematopoietic cells are characterized by a genomewide undermethylation dip around the transcription start site and a hierarchical epigenetic plasticity. Blood 114:4968-78 . doi: 10.1182/blood-2009-01-197780 
Cimmino L, Dolgalev I, Wang Y, et al (2017) Restoration of TET2 Function Blocks Aberrant Self-Renewal and Leukemia Progression. Cell 170:1079-1095.e20 . doi: 10.1016/j.cell.2017.07.032

Clappier E, Gerby B, Sigaux F, et al (2011) Clonal selection in xenografted human T cell acute lymphoblastic leukemia recapitulates gain of malignancy at relapse. J Exp Med 208:653-661 . doi: 10.1084/jem.20110105

Clatterbuck H, Sober E, Lewontin R (2013) Selection never dominates drift (nor vice versa). Biol Philos 28:577592 . doi: 10.1007/s10539-013-9374-2

de Bruin EC, McGranahan N, Mitter R, et al (2014) Spatial and temporal diversity in genomic instability processes defines lung cancer evolution. Science (80- ) 346:251-256 . doi: 10.1126/science.1253462

Ding L, Ley TJ, Larson DE, et al (2012) Clonal evolution in relapsed acute myeloid leukaemia revealed by wholegenome sequencing. Nature 481:506-10 . doi: 10.1038/nature10738

Dong L, Yu W-M, Zheng H, et al (2016) Leukaemogenic effects of Ptpn11 activating mutations in the stem cell microenvironment. Nature 539:304-308 . doi: 10.1038/nature20131

Fabian A, Barok M, Vereb G, Szollosi J (2009) Die hard: are cancer stem cells the Bruce Willises of tumor biology? Cytom A 75:67-74 . doi: 10.1002/cyto.a.20690

Francis JM, Zhang C-Z, Maire CL, et al (2014) EGFR variant heterogeneity in glioblastoma resolved through singlenucleus sequencing. Cancer Discov 4:956-71 . doi: 10.1158/2159-8290.CD-13-0879

Gao R, Davis A, McDonald TO, et al (2016) Punctuated copy number evolution and clonal stasis in triple-negative breast cancer. Nat Genet 48:1119-30 . doi: 10.1038/ng.3641

Genovese G, Kahler AK, Handsaker RE, et al (2014) Clonal hematopoiesis and blood-cancer risk inferred from blood DNA sequence. N Engl J Med 371:2477-2487 . doi: 10.1056/NEJMoa1409405

Gerlinger M, Horswell S, Larkin J, et al (2014) Genomic architecture and evolution of clear cell renal cell carcinomas defined by multiregion sequencing. Nat Genet 46:225-33 . doi: 10.1038/ng.2891

Gerlinger M, Rowan AJ, Horswell S, et al (2012) Intratumor heterogeneity and branched evolution revealed by multiregion sequencing. N Engl J Med 366:883-92 . doi: 10.1056/NEJMoa1113205

Germain P-L (2012) Cancer cells and adaptive explanations. Biol Philos 27:785-810 . doi: 10.1007/s10539-0129334-2

Germain PL, Laplane L (2017) Metastasis as supra-cellular selection? A reply to Lean and Plutynski. Biol Philos 32:281-287 . doi: 10.1007/s10539-016-9555-x

Godfrey-Smith P (2009) Darwinian Populations and Natural Selection. Oxford University Press, Oxford

Greaves M (2010) Cancer stem cells: back to Darwin? Semin Cancer Biol 20:65-70 . doi: S1044-579X(10)00010-6 [pii]10.1016/j.semcancer.2010.03.002

Greaves M (2013) Cancer stem cells as “units of selection." Evol Appl 6:102-108 . doi: 10.1111/eva.12017

Greaves M (2007) Darwinian medicine: a case for cancer. Nat Rev Cancer 7:213-221 . doi: 10.1038/nrc2071

Greaves M (2015) Evolutionary determinants of cancer. Cancer Discov 5:806-20 . doi: 10.1158/2159-8290.CD15-0439

Greaves M, Maley CC (2012) Clonal evolution in cancer. Nature 481:306-313 . doi: nature10762 [pii] $10.1038 /$ nature10762

Hirsch P, Mamez AC, Belhocine R, et al (2016) Clonal history of a cord blood donor cell leukemia with prenatal 
somatic JAK2 V617F mutation. Leukemia 30:1756-1759 . doi: 10.1038/leu.2016.31

Hou Y, Song L, Zhu P, et al (2012) Single-cell exome sequencing and monoclonal evolution of a JAK2-negative myeloproliferative neoplasm. Cell 148:873-85 . doi: 10.1016/j.cell.2012.02.028

Hughes AEO, Magrini V, Demeter R, et al (2014) Clonal architecture of secondary acute myeloid leukemia defined by single-cell sequencing. PLoS Genet 10:e1004462 . doi: 10.1371/journal.pgen.1004462

Itzykson R, Kosmider O, Renneville A, et al (2013) Clonal architecture of chronic myelomonocytic leukemias. Blood 121:2186-2198 . doi: 10.1182/blood-2012-06-440347

Jaiswal S, Fontanillas P, Flannick J, et al (2014) Age-related clonal hematopoiesis associated with adverse outcomes. N Engl J Med 371:2488-2498 . doi: 10.1056/NEJMoa1408617

Jan M, Snyder TM, Corces-Zimmerman MR, et al (2012) Clonal evolution of preleukemic hematopoietic stem cells precedes human acute myeloid leukemia. Sci Transl Med 4:149ra118 . doi: 4/149/149ra118 [pii] 10.1126/scitransImed.3004315

Kim TH, Yoshida K, Kim YK, et al (2015) Clonal dynamics in a single AML case tracked for 9 years reveals the complexity of leukemia progression. Leukemia 30:295-302 . doi: 10.1038/leu.2015.264

Koren S, Reavie L, Couto JP, et al (2015) PIK3CA(H1047R) induces multipotency and multi-lineage mammary tumours. Nature 525:114-118 . doi: 10.1038/nature14669

Kreso A, Dick JE (2014) Evolution of the cancer stem cell model. Cell Stem Cell 14:275-91 . doi: S19345909(14)00057-5 [pii]10.1016/j.stem.2014.02.006

Lagasse E (2008) Cancer stem cells with genetic instability: the best vehicle with the best engine for cancer. Gene Ther 15:136-142 . doi: 3303068 [pii]10.1038/sj.gt.3303068

Landau DA, Carter SL, Stojanov P, et al (2013) Evolution and impact of subclonal mutations in chronic lymphocytic leukemia. Cell 152:714-26 . doi: 10.1016/j.cell.2013.01.019

Lang F, Wojcik B, Rieger MA (2015) Stem Cell Hierarchy and Clonal Evolution in Acute Lymphoblastic Leukemia. Stem Cells Int 2015:137164 . doi: 10.1155/2015/137164

Laplane L (2015) Reprogramming and Stemness. Perspect Biol Med 58:229-46 . doi: 10.1353/pbm.2015.0022

Laplane L (2016) Cancer Stem Cells: Philosophy and Therapies. Harvard Univerity Press, Cambridge, MA

Laplane L (2014) Identifying theories in developmental biology. The case of the cancer stem cell theory. In: Minelli A, Pradeu T (eds) Toward a theory of development. Oxford University Press, Oxford, pp 246-259

Latil M, Nassar D, Beck B, et al (2017) Cell-Type-Specific Chromatin States Differentially Prime Squamous Cell Carcinoma Tumor-Initiating Cells for Epithelial to Mesenchymal Transition. Cell Stem Cell 20:191-204.e5 . doi: 10.1016/j.stem.2016.10.018

Lean C, Plutynski A (2016) The evolution of failure: explaining cancer as an evolutionary process. Biol Philos 31:39-57 . doi: 10.1007/s10539-015-9511-1

Lewontin RC (1970) The Units of Selection. Annu Rev Ecol Syst 1:1-18 . doi: 10.1146/annurev.es.01.110170.000245

Lewontin RC (1978) Adaptation. Sci Am 239:212-8, 220, 222 passim

Ling S, Hu Z, Yang Z, et al (2015) Extremely high genetic diversity in a single tumor points to prevalence of nonDarwinian cell evolution. Proc Natl Acad Sci 112:E6496-6505 . doi: 10.1073/pnas.1519556112 
Lipinski KA, Barber LJ, Davies MN, et al (2016) Cancer Evolution and the Limits of Predictability in Precision Cancer Medicine. Trends in cancer 2:49-63 . doi: 10.1016/j.trecan.2015.11.003

Mack SC, Witt H, Piro RM, et al (2014) Epigenomic alterations define lethal CIMP-positive ependymomas of infancy. Nature 506:445-50 . doi: 10.1038/nature13108

Markowetz F (2016) A saltationist theory of cancer evolution. Nat Genet 48:1102-3 . doi: 10.1038/ng.3687

Martincorena I, Raine KM, Gerstung M, et al (2017) Universal Patterns of Selection in Cancer and Somatic Tissues. Cell 171:1029-1041.e21

Martincorena I, Roshan A, Gerstung M, et al (2015) High burden and pervasive positive selection of somatic mutations in normal human skin. Science (80- ) 348:880-886 . doi: 10.1126/science.aaa6806

Medema JP (2017) Targeting the Colorectal Cancer Stem Cell. N Engl J Med 377:888-890 . doi: 10.1056/NEJMcibr1706541

Melchor L, Brioli A, Wardell CP, et al (2014) Single-cell genetic analysis reveals the composition of initiating clones and phylogenetic patterns of branching and parallel evolution in myeloma. Leukemia 28:1705-15 . doi: 10.1038/leu.2014.13

Merlevede J, Droin N, Qin T, et al (2016) Mutation allele burden remains unchanged in chronic myelomonocytic leukaemia responding to hypomethylating agents. Nat Commun 7:10767 . doi: 10.1038/ncomms10767

Merlo LM, Pepper JW, Reid BJ, Maley CC (2006) Cancer as an evolutionary and ecological process. Nat Rev Cancer 6:924-935 . doi: nrc2013 [pii]10.1038/nrc2013

Meyer M, Reimand J, Lan X, et al (2015) Single cell-derived clonal analysis of human glioblastoma links functional and genomic heterogeneity. Proc Natl Acad Sci U S A 112:851-6 . doi: 10.1073/pnas.1320611111

Mikkelsen TS, Hanna J, Zhang X, et al (2008) Dissecting direct reprogramming through integrative genomic analysis. Nature 454:49-55 . doi: 10.1038/nature07056

Murugaesu N, Wilson GA, Birkbak NJ, et al (2015) Tracking the genomic evolution of esophageal adenocarcinoma through neoadjuvant chemotherapy. Cancer Discov 5:821-31 . doi: 10.1158/2159-8290.CD-15-0412

Nassar D, Blanpain C (2016) Cancer Stem Cells: Basic Concepts and Therapeutic Implications. Annu Rev Pathol 11:47-76 . doi: 10.1146/annurev-pathol-012615-044438

Navin N, Kendall J, Troge J, et al (2011) Tumour evolution inferred by single-cell sequencing. Nature 472:90-4 . doi: $10.1038 /$ nature09807

Nik-Zainal S, Alexandrov LB, Wedge DC, et al (2012a) Mutational processes molding the genomes of 21 breast cancers. Cell 149:979-93 . doi: 10.1016/j.cell.2012.04.024

Nik-Zainal S, Van Loo P, Wedge DC, et al (2012b) The life history of 21 breast cancers. Cell 149:994-1007 . doi: 10.1016/j.cell.2012.04.023

Notta F, Mullighan CG, Wang JC, et al (2011) Evolution of human BCR-ABL1 lymphoblastic leukaemia-initiating cells. Nature 469:362-367 . doi: nature09733 [pii] 10.1038/nature09733

Odoux C, Fohrer H, Hoppo T, et al (2008) A stochastic model for cancer stem cell origin in metastatic colon cancer. Cancer Res 68:6932-6941 . doi: 68/17/6932 [pii]10.1158/0008-5472.CAN-07-5779

Ortmann CA, Kent DG, Nangalia J, et al (2015) Effect of mutation order on myeloproliferative neoplasms. N EngI J Med 372:601-12 . doi: 10.1056/NEJMoa1412098 
Pantic I (2011) Cancer stem cell hypotheses: impact on modern molecular physiology and pharmacology research. J Biosci 36:957-961

Pepper JW, Scott Findlay C, Kassen R, et al (2009) Cancer research meets evolutionary biology. Evol Appl 2:6270 . doi: $10.1111 /$ j.1752-4571.2008.00063.x

Pepper JW, Sprouffske K, Maley CC (2007) Animal cell differentiation patterns suppress somatic evolution. PLoS Comput Biol 3:e250 . doi: 10.1371/journal.pcbi.0030250

Piccirillo SG, Combi R, Cajola L, et al (2009) Distinct pools of cancer stem-like cells coexist within human glioblastomas and display different tumorigenicity and independent genomic evolution. Oncogene 28:1807-1811 . doi: onc200927 [pii] 10.1038/onc.2009.27

Polyak K (2007) Breast cancer: origins and evolution. J Clin Invest 117:3155-3163 . doi: 10.1172/JCI33295

Potter NE, Ermini L, Papaemmanuil E, et al (2013) Single-cell mutational profiling and clonal phylogeny in cancer. Genome Res 23:2115-25 . doi: 10.1101/gr.159913.113

Quintana E, Shackleton M, Sabel MS, et al (2008) Efficient tumour formation by single human melanoma cells. Nature 456:593-598 . doi: nature07567 [pii]10.1038/nature07567

Rausch T, Jones DTW, Zapatka M, et al (2012) Genome sequencing of pediatric medulloblastoma links catastrophic DNA rearrangements with TP53 mutations. Cell 148:59-71 . doi: 10.1016/j.cell.2011.12.013

Reya T, Morrison SJ, Clarke MF, Weissman IL (2001) Stem cells, cancer, and cancer stem cells. Nature 414:10511 . doi: $10.1038 / 3510216735102167$ [pii]

Reynaud D, Pietras E, Barry-Holson K, et al (2011) IL-6 controls leukemic multipotent progenitor cell fate and contributes to chronic myelogenous leukemia development. Cancer Cell 20:661-73 . doi: 10.1016/j.ccr.2011.10.012

Schuh A, Becq J, Humphray S, et al (2012) Monitoring chronic lymphocytic leukemia progression by whole genome sequencing reveals heterogeneous clonal evolution patterns. Blood 120:4191-6 . doi: 10.1182/blood-2012-05-433540

Shackleton M, Quintana E, Fearon ER, Morrison SJ (2009) Heterogeneity in cancer: cancer stem cells versus clonal evolution. Cell 138:822-829 . doi: S0092-8674(09)01030-7 [pii] 10.1016/j.cell.2009.08.017

Shain AH, Yeh I, Kovalyshyn I, et al (2015) The Genetic Evolution of Melanoma from Precursor Lesions. N Engl J Med 373:1926-36 . doi: 10.1056/NEJMoa1502583

Shipitsin M, Campbell LL, Argani P, et al (2007) Molecular definition of breast tumor heterogeneity. Cancer Cell 11:259-273 . doi: S1535-6108(07)00029-3 [pii]10.1016/j.ccr.2007.01.013

Siegmund KD, Marjoram P, Tavaré S, Shibata D (2009) Many colorectal cancers are "flat" clonal expansions. Cell Cycle 8:2187-93

Simons BD (2016) Deep sequencing as a probe of normal stem cell fate and preneoplasia in human epidermis. Proc Natl Acad Sci U S A 113:128-33 . doi: 10.1073/pnas.1516123113

Sottoriva A, Barnes CP, Graham TA (2017) Catch my drift? Making sense of genomic intra-tumour heterogeneity. Biochim Biophys Acta - Rev Cancer. doi: 10.1016/j.bbcan.2016.12.003

Sottoriva A, Kang H, Ma Z, et al (2015) A Big Bang model of human colorectal tumor growth. Nat Genet 47:209216 . doi: $10.1038 / \mathrm{ng} .3214$ 
Sottoriva A, Spiteri I, Piccirillo SGM, et al (2013) Intratumor heterogeneity in human glioblastoma reflects cancer evolutionary dynamics. Proc Natl Acad Sci U S A 110:4009-14 . doi: 10.1073/pnas.1219747110

Sottoriva A, Verhoeff JJ, Borovski T, et al (2010) Cancer stem cell tumor model reveals invasive morphology and increased phenotypical heterogeneity. Cancer Res 70:46-56 . doi: 0008-5472.CAN-09-3663 [pii] 10.1158/0008-5472.CAN-09-3663

Sprouffske K, Athena Aktipis C, Radich JP, et al (2013) An evolutionary explanation for the presence of cancer nonstem cells in neoplasms. Evol Appl 6:92-101 . doi: 10.1111/eva.12030

Stephens PJ, Greenman CD, Fu B, et al (2011) Massive genomic rearrangement acquired in a single catastrophic event during cancer development. Cell 144:27-40 . doi: S0092-8674(10)01377-2 [pii]10.1016/j.cell.2010.11.055

Sun J, Ramos A, Chapman B, et al (2014) Clonal dynamics of native haematopoiesis. Nature 514:322-327 . doi: $10.1038 /$ nature13824

Suzuki M, Harashima A, Okochi A, et al (2004) 5-Azacytidine supports the long-term repopulating activity of cord blood CD34(+) cells. Am J Hematol 77:313-5 . doi: 10.1002/ajh.20178

Takebe N, Ivy SP (2010) Controversies in cancer stem cells: targeting embryonic signaling pathways. Clin Cancer Res 16:3106-3112 . doi: 1078-0432.CCR-09-2934 [pii]10.1158/1078-0432.CCR-09-2934

Thirlwell C, Will OCC, Domingo E, et al (2010) Clonality assessment and clonal ordering of individual neoplastic crypts shows polyclonality of colorectal adenomas. Gastroenterology 138:1441-54, 1454-7 . doi: 10.1053/j.gastro.2010.01.033

Tomasetti C, Li L, Vogelstein B (2017) Stem cell divisions, somatic mutations, cancer etiology, and cancer prevention. Science (80- ) 355:1330-1334 . doi: 10.1126/science.aaf9011

Tomasetti C, Vogelstein B (2015) Variation in cancer risk among tissues can be explained by the number of stem cell divisions. Science (80- ) 347:78-81 . doi: 10.1126/science.1260825

Van Keymeulen A, Lee MY, Ousset M, et al (2015) Reactivation of multipotency by oncogenic PIK3CA induces breast tumour heterogeneity. Nature 525:119-23 . doi: 10.1038/nature14665

Walter MJ, Shen D, Ding L, et al (2012) Clonal architecture of secondary acute myeloid leukemia. N Engl J Med 366:1090-8 . doi: 10.1056/NEJMoa1106968

Welner RS, Amabile G, Bararia D, et al (2015) Treatment of chronic myelogenous leukemia by blocking cytokine alterations found in normal stem and progenitor cells. Cancer Cell 27:671-81 . doi: 10.1016/j.ccell.2015.04.004

Wicha MS, Liu S, Dontu G (2006) Cancer stem cells: an old idea--a paradigm shift. Cancer Res 66:1883-1886 . doi: 66/4/1883 [pii]10.1158/0008-5472.CAN-05-3153

Williams MJ, Werner B, Barnes CP, et al (2016) Identification of neutral tumor evolution across cancer types. Nat Genet 48:238-244 . doi: 10.1038/ng.3489

Xie M, Lu C, Wang J, et al (2014) Age-related mutations associated with clonal hematopoietic expansion and malignancies. Nat Med 20:1472-8 . doi: 10.1038/nm.3733

Xu X, Hou Y, Yin X, et al (2012) Single-cell exome sequencing reveals single-nucleotide mutation characteristics of a kidney tumor. Cell 148:886-95 . doi: 10.1016/j.cell.2012.02.025 
Yachida S, Wood LD, Suzuki M, et al (2016) Genomic Sequencing Identifies ELF3 as a Driver of Ampullary Carcinoma. Cancer Cell 29:229-240 . doi: 10.1016/j.ccell.2015.12.012

Zhang J, Fujimoto J, Zhang J, et al (2014) Intratumor heterogeneity in localized lung adenocarcinomas delineated by multiregion sequencing. Science 346:256-9 . doi: 10.1126/science.1256930

Zhang XC, Xu C, Mitchell RM, et al (2013) Tumor evolution and intratumor heterogeneity of an oropharyngeal squamous cell carcinoma revealed by whole-genome sequencing. Neoplasia 15:1371-8 